\title{
Total Hip Arthroplasty in 2017 - Current Concepts and Recent Advances
}

Total hip arthroplasty (THA) is one of the most successful interventions not only in orthopedics but also in modern medicine. Since its first implantation in the 1950s, improvements in surgical technique, technology, and postoperative rehabilitation have greatly increased the effectiveness and success of this procedure.

However, the burden of hip arthritis is on the rise and it is estimated that more than 950,000 primary and revision THAs were performed globally in $2010 .{ }^{1}$ The profile of the patients having their hips replaced is changing as well from the elderly with minimal needs to the young adult who wishes to have the maximum from their hip. Surgeons performing a THA are therefore presented with a unique challenge of meeting high patient expectations and ensuring excellent outcomes and at the same time using the most evidence-based and cost-effective implants and bearing surface technology. Furthermore, the current health economic environment worldwide has made it more challenging to deliver this, especially in a public system.

Therefore, if hip surgeons are focused on continual improvement of functional outcome of this already successful procedure and also the longevity of prosthesis, it is essential that they are aware of the advances in surgical technique and evidence behind the choice of implants. Moreover, a good overview of the management of complications such as adverse reaction to metal debris (ARMD) and infection is required. Finally, an understanding of basic science behind THA including biomechanics as well as how to assess performance and to measure functional outcome following a THA is indispensable.

This symposium addresses several key topics mentioned above, right from the basics to the most current and topical debates involving a THA.

Houcke et al. ${ }^{2}$ present key concepts in biomechanics of the hip and their application to THA, which has played a crucial and integral role in achieving excellent outcomes. As a surgeon, one can be too focused on the surgical technique and miss out on the biomechanical principles on which a THA is based, and this article provides a good account of the history and biomechanical development of the THA. Reading an article on biomechanics for any orthopedic surgeon could be a tough task, but this one is concise, informative, interesting, and fairly easy to read.

Our next article focuses on approaches to the hip. ${ }^{3}$ The minimally invasive anterior hip approach is quite popular currently and has its advocates. Moretti et al. ${ }^{3}$ describe the three most popular surgical approaches for THA. The article also highlights the history and technique for each of these approaches and provides a review of the outcomes and complications for each approach. Although my personal favorite is the posterior approach which has also been reported as the most common surgical approach used worldwide for a THA, each of the other two approaches, i.e., the direct lateral and the direct anterior approach, has their own strengths and also weaknesses! The authors mention that scientifically robust studies comparing these approaches are still lacking, and they recommend surgeons to choose whichever approach they are most comfortable with. Meanwhile, whilst evidence is still lacking, I plan to stand by the approach that I am most familiar with!

Maggs et al. ${ }^{4}$ from Exeter, UK (the home of the cemented THA), discuss relative merits of the cemented and uncemented prostheses in THA by examining the designs of different types of prostheses and describing the history behind their development and the reported results. The authors' mention their preference for the cemented technique and explain the reasoning and concepts behind this preference (there may be an element of bias here for obvious reasons). Their article also covers the history of a cemented THA, and optimum cementing technique, which I think is very informative and educational and a must read for every hip surgeon.

Acetabular component positioning is the most important determinant in terms of reducing the risk of dislocation following a THA. Bhaskar et al. ${ }^{5}$ discuss the current concepts in acetabular positioning in a THA. They aim to provide a systematic and pragmatic approach in achieving the best position in individual cases by providing the evidence on acetabular component positioning. Whilst twodimensional (2D) templating is the most common method used, this does have limitations. The article makes very interesting reading and I would encourage you to have a closer look at "How to achieve target depth?," "How to achieve target height?," and "How to achieve target angular position?" if you wish to achieve some more confidence in your acetabular component positioning technique. The tips discussed in the article may help you to reconstruct a 3D image of each patient's acetabular anatomy whilst positioning the acetabular component.

Lopez et al. ${ }^{6}$ who leads the Bone Infection Service in Cambridge, UK, provides a comprehensive update on the management of the infected THA, which is one of the most devastating complications for the patient as well as for the surgeon. Are you familiar with interleukin- 6 as a serum marker for infection, or the alpha-defensin test on the synovial fluid for the diagnosis of an infected THA? How about indium-111-labeled white cell scan which can 
be performed, to precisely identify the site of the infection within the hip and their sensitivity? If not, then this article provides you with all the latest developments in this arena. The economic consequences associated with treating periprosthetic infections are also substantial. I would dread to think that it costs almost similar to buy a BMW Series 1 car and to perform a revision THA secondary to infection!

Malviya et al. ${ }^{7}$ discuss the various sources of information that hip surgeons can reliably refer to while assessing outcomes following a THA. They also use these sources to discuss the controversies in the literature pertaining to the best method of fixation, the best bearing surface, and the optimum size of the femoral head. There is too much data in this article, and there is a tendency to get lost! If that happens, then I would advise you to look at "Recommendation" at the end of each section in the results of the article to grasp the verdict.

Finally, we discuss a strategy for the followup of patients with metal-on-metal (MoM) hips by comparing and contrasting the current guidelines for managing patients with MoM hips and the established risk factors for ARMD. Evidence regarding the optimum management of patients with MoM hips and the indications for revision surgery are also discussed. We have also tried to address several difficult questions which frequently bother the practicing hip surgeons - "Which patients are at risk of ARMD?," "What is the evidence base for the frequency of followup?," "What is the threshold for concern with metal ion levels?," "When should revision surgery be considered and conducted?," etc., I hope this review will come in handy when you manage your patients with MoM hips.

Hip surgeons from specialist centers around the globe have contributed to this issue and we have tried to address most issues which are relevant in our clinical practice. I hope these articles will help you reflect and determine what is truly best for your patients and what works in your circumstances, as well as facilitate our drive to further advance the field of hip arthroplasty surgery.

We have had a great and tedious time in putting this symposium together and I thoroughly recommend all the articles to you. I hope you enjoy reading it and refreshing your knowledge.
Vikas Khanduja

Department of Trauma and Orthopaedics, Addenbrooke's Hospital, Cambridge University Hospitals NHS Foundation Trust, Cambridge, $C B 20 Q Q, U K$

Address for correspondence: Mr. Vikas Khanduja, Department of Trauma and Orthopaedics, Addenbrooke's Hospital, Cambridge University Hospitals NHS Foundation Trust, Hills Road, Cambridge, CB2 OQQ, UK.

E-mail:vk279@cam.ac.uk

\section{References}

1. Kurtz SM, Röder C, Lau E, Ong K, Widmer M, Maravic M, et al. International Survey of Primary and Revision Total Hip Replacement. $56^{\text {th }}$ Annual Meeting of the Orthopaedic Research Society; 2010.

2. Houcke JV, Khanduja V, Pattyn C, Audenaert E. The history of biomechanics in total hip arthroplasty. Indian J Orthop 2017;51:359-67.

3. Moretti VM, Zachary DP. Surgical approaches for total hip arthroplasty. Indian J Orthop 2017;51:368-76.

4. Maggs J, Wilson M. The relative merits of cemented and uncemented prostheses in total hip arthroplasty. Indian J Orthop 2017;51:377-85.

5. Bhaskar D, Rajpura A, Board T. Current concepts in acetabular positioning in total hip arthroplasty. Indian J Orthop 2017;51:386-96.

6. Lopez D, Leach I, Moore E, Norrish AR. Management of the infected total hip arthroplasty. Indian J Orthop 2017;51:397-404.

7. Malviya A, Abdul N, Khanduja V. Outcomes following total hip arthroplasty: A review of the registry data. Indian J Orthop 2017;51:405-13.

This is an open access article distributed under the terms of the Creative Commons Attribution-NonCommercial-ShareAlike 3.0 License, which allows others to remix, tweak, and build upon the work non-commercially, as long as the author is credited and the new creations are licensed under the identical terms.

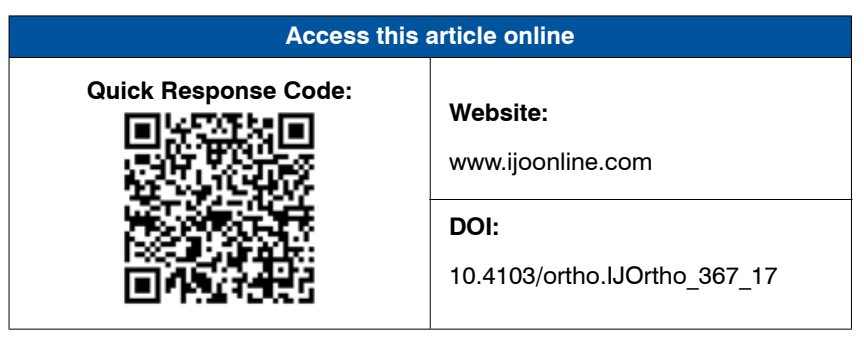

How to cite this article: Khanduja V. Total hip arthroplasty in $2017-$ Current concepts and recent advances. Indian J Orthop 2017;51:357-8. 